\title{
Efecto comparativo de la Actividad Física Aeróbica y los Dispositivos ortopédicos estabilizadores en el dolor orofacial de origen musculoesqueletal en individuos con Trastornos temporomandibulares
}

Comparative effect of aerobic physical activity and stabilizing orthopedic devices on orofacial pain of musculoskeletal origin in individuals with temporomandibular disorders.

Efeito comparativo da atividade física aeróbica e dispositivos ortopédicos estabilizadores na dor orofacial de origem musculoesquelética em indivíduos com disfunção temporomandibular

\author{
Luis Andrés Rodríguez Figueroa' ORCID: 0000-0003-2130-4575 \\ Lucia Stefanelli² ORCID: 0000-0003-0651-6959 \\ Raul Riva Bernasconi' ORCID: 0000-0002-8701-9286
}

DOI: 10.22592/ode2020n36a3

\section{Resumen}

El presente estudio evaluó la eficacia comparativa entre la actividad física aeróbica (AFA) y los dispositivos ortopédicos estabilizadores(DOE) en el manejo del dolor orofacial de origen músculo esqueletal en individuos con trastornos temporomandibulares (TTM).

Participaron voluntarios dentados bimaxilar con dolor de origen músculo esqueletal con edades comprendidas entre 18 y 40 años. Para la evaluación de la variable dolor el instrumento de medición fue la escala verbal análoga. Cada uno de los grupos de tratamientos estuvo compuesto por siete individuos, los cuales fueron evaluados durante ocho (AFA) y seis (DOE) semanas respectivamente.

La AFA se caracterizó por un descenso semanal significativo de 0.155 puntos en la medición del dolor. En tanto con DOE la tendencia fue aún más pronunciada que en el tratamiento con AFA, siendo esta diferencia significativa.

El presente estudio demostró que, tanto la intervención con DOE como con AFA contribuyeron a la disminución del dolor orofacial de origen músculo esqueletal en voluntarios con TTM.

Palabras clave: Trastornos temporomandibulares, dolor muscoloesqueletal, ejercicio aeróbico, dispositivos oclusales.

Cátedra de Rehabilitación, prostodoncia fija y trastornos temporomandibulares, Facultad de Odontología, Universidad de la República, Montevideo, Uruguay.

2 Departamento de educación física y salud, Instituto Superior de Educación Física, Universidad de la República, Montevideo, Uruguay.

Fecha de recibido: 4/5/2020 - Fecha de aceptado: 14/9/2020 


\begin{abstract}
The present study evaluated the efficacy of aerobic physical activity (AFA) in the management of orofacial pain of skeletal muscle origin in individuals with temporomandibular disorders compared to treatment with a stabilizing orthopedic device (DOE). Volunteers with skeletal muscle pain aged between 18 and 40 years and bimaxillary teeth were involved. For the evaluation of the pain variable, the measurement instrument was the analog verbal scale. Each of the treatment groups was composed of seven individuals, who were evaluated for eight (AFA) and six (DOE) weeks, respectively.

AFA was characterized by a significant weekly decrease of 0.155 points in pain measurement. As for DOE, the trend was even more pronounced than in the AFA treatment, this difference being significant. The present study demonstrated that both DOE and AFA intervention contributed to the reduction of musculoskeletal orofacial pain in TTM volunteers.
\end{abstract}

Keywords: Temporomandibular disorders, musculoskeletal pain, aerobic exercise, occlusal devices.

\section{Introducción}

Los trastornos témporomandibulares (TTM) constituyen un grupo heterogéneo de patologías que afectan a las articulaciónes temporomandibulares (ATM), los músculos masticatorios o a ambos $^{(1)}$. Para el glosario de términos prostodóncicos son las condiciones que producen una función anormal, incompleta o alterada de las ATM y/o los músculos de la masticación ${ }^{(2)}$.El dolor en la región temporomandibular parece ser relativamente común y ocurre en aproximadamente el $10 \%$ de la población mayor de 18

\section{Resumo}

O presente estudo avaliou a eficácia da atividade física aeróbica (AFA) no tratamento da dor orofacial de origem muscular esquelética em indivíduos com disfunção temporomandibular em comparação ao tratamento com dispositivo ortopédico estabilizador (DOE). Participaram voluntários com dor muscular esquelética com idade entre 18 e 40 anos e dentes bimaxilares. Para a avaliação da variável dor, o instrumento de medida foi a escala verbal analógica. Cada um dos grupos de tratamento foi composto por sete indivíduos, avaliados por oito (AFA) e seis (DOE) semanas, respectivamente.

AFA foi caracterizada por uma diminuição semanal significativa de 0,155 pontos na mediçáo da dor. Quanto ao DOE, a tendência foi ainda mais acentuada do que no tratamento com AFA, sendo essa diferença significativa.

O presente estudo demonstrou que a intervenção do DOE e do AFA contribuiu para a redução da dor musculoesquelética orofacial em voluntários com TTM.

Palavras-chave: Distúrbios temporomandibulares, dor musculoesquelética, exercício aeróbico, dispositivos oclusais.

años; es principalmente una afección de adultos jóvenes, de mediana edad y es, aproximadamente, dos veces más común en mujeres que en hombres ${ }^{(3)}$.

Los TTM son afecciones que presentan una alta prevalencia a nivel de la población general. La prevalencia de signos en un estudio realizado en una población europea es de $34,5 \%{ }^{(4)}$. Otro estudio realizado en Australia mostró una prevalencia de síntomas de TTM de 77,2\% en una cohorte de estudiantes de odontología ${ }^{(5)}$. En Uruguay, el 55\% de la población presenta 
por lo menos un síntoma y el $44 \%$ de la población por lo menos un signo ${ }^{(6)}$. Estos estudios también concluyen en una mayor prevalencia en mujeres.

Resulta importante poder identificar los principales factores causales que pueden llevar a padecer un TTM, ya que permitiría seleccionar el tratamiento más apropiado y efectivo para cada paciente. Los tratamientos reversibles más utilizados para el síntoma dolor incluyen: terapia con dispositivos ortopédicos estabilizadores (DOE), farmacoterapia, fisioterapia, sicoterapia (estrategias conductuales), etc.; las que pueden ser indicadas en forma individual o combinada ${ }^{(7)}$.

Los DOE han demostrado tener evidencia suficiente para su empleo en el tratamiento de la mialgia y la artralgia del sistema masticatorio (8). Sin embargo, su mecanismo de acción no es claro y aún es motivo de controversia.

Diversos problemas metodológicos relativos a los diseños de investigación han dificultado la comprensión de la real eficacia y los mecanismos de acción de los DOE. Por ejemplo, tamaño pequeño de la muestra, cegamientos inadecuados y cortos tiempos de seguimiento constituyen algunos de los problemas detectados en trabajos previos ${ }^{(9)}$.

Las medidas terapéuticas que han demostrado ser más eficaces para modificar la actitud ante el dolor y el estrés son la actividad física de tipo aeróbico (AFA) y la terapia manual ${ }^{(10)}$.

Estudios previos han demostrado que las intervenciones con AFA tienen resultados positivos para la disminución del dolor en el tratamiento de la fibromialgia ${ }^{(11-13)}$.

Hasta el momento no se han realizado estudios para evaluar si la AFA es efectiva para la disminución de dolor en pacientes con dolor muscular por TTM.

La betaendorfina (BE) es una hormona, un opioide endógeno, secretado por la glándula anterior de la hipófisis. Entre sus efectos más importantes se destaca la analgesia, mayor tolerancia al lactato y disminución del disconfort muscular. ${ }^{(14)}$ Aumentan en el torrente sanguíneo durante la AFA prolongada, produciendo una disminución del síntoma doloroso. En comparación con las encefalinas presenta mayor resistencia a la degradación enzimatica ${ }^{(15)}$. Valim, referenciando a otros autores, sostiene que la AFA tiene influencia en el sistema serotoninérgico, aumenta la actividad simpática, mejora el sueńo y promueve un sentimiento de bien estar psicológico ${ }^{(11)}$.

El presente estudio evaluó la eficacia de la AFA en el manejo del dolor orofacial de origen músculo esqueletal en individuos con TTM, comparando con tratamiento con DOE.

\section{Materiales y métodos}

\section{Material}

Se trató de un estudio clínico, comparativo, prospectivo, longitudinal, controlado y aleatorizado.

La muestra fue seleccionada entre estudiantes de las carreras de la Facultad de Odontología, Universidad de la Republica, Uruguay. Incluyo 14 voluntarios mujeres. El método de reclutamiento fue consecutivo y se utilizó un llamado público en el año 2016, dirigido a voluntarios que padecieran dolor orofacial.

Aspectos éticos: El proyecto fue aprobado por el comité de Ética de la Facultad de Odontología de la Universidad de la República (Expediente 249/15).

Los voluntarios firmaron consentimiento informado antes de su participación.

Criterios de Inclusión: voluntarios con dolor de origen músculo esqueletal por TTM, con edad comprendida entre 18 y 40 años, dentados bi maxilar y que presentaran comprobante de aptitud física expedido por institución competente.

Criterios de exclusión: voluntarios con: dolor de otro origen al músculo esqueletal por TTM, embarazadas, que desarrollen actividad física aeróbica habitual, portadores de DOE al momento de la intervención, afectaciones respira- 
torias crónicas y/o agudas, alteraciones psiquiátricas severas, enfermedades cardiacas inestables o no tratadas y con antecedente de infarto de miocardio en los pasados 6 meses.

Criterios de eliminación: aquellos que no cumplieron con al menos el $80 \%$ de las sesiones de AFA, así como los pacientes que no cumplieron con las indicaciones de uso y controles de los DOE.

\section{Método}

Se realizó el examen clínico general, regional y local de los pacientes con la finalidad de verificar los criterios de inclusión-exclusión.

Se diferenció a los voluntarios con dolor orofacial por TTM de origen músculo esqueletal de los de otro origen, siguiendo los criterios del DC-TMD. ${ }^{(16)}$ y el protocolo de calibración del estudio de Riva et al de Mayo de $2011^{(6)}$. Se realizó palpación de músculos masticadores (maseteros y temporales) y de polo externo de las ATM.

\section{Variables e instrumentos de medición}

Para la evaluación de las variables dolor y estrés emocional, el instrumento de medición fue la escala verbal análoga (EVA). Para la recolección de datos se utilizó una encuesta que incluye el instrumento de medición mencionado (Fig. 1). Ésta fue utilizada en repetidas oportunidades, desde el inicio hasta el final de las intervenciones, con la finalidad de evaluar la evolución del síntoma relatado por el paciente. Cada uno de los grupos de tratamiento estuvo compuesto de siete individuos, los cuales fueron evaluados durante ocho (AFA) y seis (DOE) semanas, más una evaluación previa para determinar valores promedio al inicio del tratamiento. En cada una de las evaluaciones se registraron autoreportes de dolor y stress en tres momentos del día: mañana, tarde y noche. Para la evaluación previa se les proveyó de un diario del dolor dos semanas antes de la intervención, de manera de obtener un dato promedio de dolor de base, esto se realiza considerando las características del mismo y sus fluctuaciones.

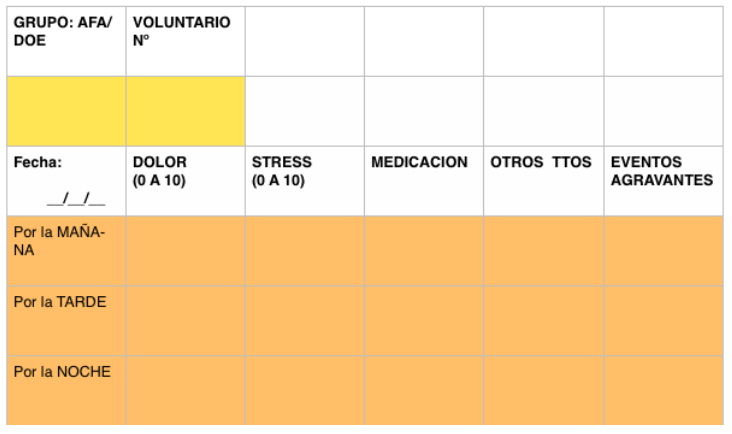

Fig. 1

\section{Intervenciones y grupos:}

La muestra se dividió de forma aleatoria en dos grupos de 7 voluntarios por cada uno.

Grupo A (GA) Individuos que realizaron exclusivamente AFA y Grupo B (GB) Individuos que se les instaló DOE.

Los DOE fueron realizados en la clínica de la Facultad de Odontología por la técnica de lámina estampada y confeccionados por especialista en la temática considerando los criterios de oclusión ideal.

La AFA fue supervisada por el Instituto Superior de Educación Física (ISEF) de la Universidad de la Republica a través de estudiantes avanzados y docentes. El tipo de AFA fue Fitness aeróbica en sesiones de 60 minutos. El inicio de cada una fue con una entrada en calor de 5 a $10 \mathrm{~min}$, seguido de ejercicios aeróbicos de moderada intensidad, que no superó el 10 a $30 \%$ del $\mathrm{VO}^{2} \max 14$; la parte principal con ejercicios planificados y la vuelta a la calma, en la cual se mantiene la actividad aeróbica, a una intensidad de baja a moderada (5 a 10 minutos), con un enfoque de progresión del ejercicio $^{(17)}$.

Debido a las limitante del estudio, la forma de asegurarnos la liberación de BE fue utilizando un medidor de frecuencia cardiaca (Oximetro de pulso Advanced 3 Müller) y aplicando la fórmula de Karvonen, introducir los datos y obtener el 
nivel de consumo de oxigeno. Para considerarse asociado a la liberación de $\mathrm{BE}$ debe ser entre el 60 y $85 \%{ }^{(18-19)}$.

\section{Metodología estadística}

En tanto se registraron mediciones de dolor y stress en múltiples instancias (con frecuencia semanal y tres mediciones diarias) la comparación de la reducción en ambas variables (dolor y stress) se llevó a cabo mediante el uso de modelos de regresión de efectos mixtos[1] donde las inferencias se realizaron con un nivel de significación de 5\%. El software estadístico empleado fue el R[2].

Se procedió a comparar la evolución de cada grupo mediante un modelo de regresión que comparase las tendencias de cada tratamiento en cada momento del día. Cabe señalar que di- cho modelo incluyó, como variable de control, la presencia de eventos agravantes ya que esto podía sesgar las mediciones de cada individuo en cada momento del estudio. En este artículo se presentan los resultados de la variable dolor.

\section{Resultados}

\section{Variable Dolor}

Análisis Descriptivo.

En el Cuadro 1 se presentan las evoluciones promedio de ambos tratamientos. Se puede observar como el grupo AFA presentó un período mayor de seguimiento donde parece evidenciarse una tendencia descendente en los valores de dolor.

Cuadro 1. Evolución semanal de dolor según tratamiento

\begin{tabular}{|c|c|c|c|c|c|c|c|c|c|}
\hline & \multicolumn{9}{|c|}{ Semana } \\
\hline & 0 & 1 & 2 & 3 & 4 & 5 & 6 & 7 & $\mathbf{B}$ \\
\hline AFA & 4.10 & 3.70 & 4.00 & 3.57 & 4.05 & 2.05 & 3.90 & 3.52 & 2.52 \\
\hline Mañana & 4.43 & 5.00 & 4.29 & 3.14 & 3.71 & 2.43 & 4.14 & 4.14 & 3.43 \\
\hline Noche & 3.41 & 2.43 & 3.43 & 3.43 & 4.14 & 2.00 & 3.57 & 2.43 & 1.71 \\
\hline Tarde & 4.47 & 3.86 & 4.29 & 4.14 & 4.29 & 1.71 & 4.00 & 4.00 & 2.43 \\
\hline DOE & 4.51 & 3.89 & 3.17 & 2.71 & 3.35 & 2.00 & 1.86 & & \\
\hline Mañana & 4.51 & 4.67 & 3.83 & 3.14 & 3.67 & 2.57 & 2.57 & & \\
\hline Noche & 4.51 & 2.83 & 2.67 & 2.29 & 2.57 & 1.29 & 1.29 & & \\
\hline Tarde & 4.51 & 4.17 & 3.00 & 2.71 & 3.86 & 2.14 & 1.71 & & \\
\hline Tot & 4.31 & 3.79 & 3.62 & 3.14 & 3.71 & 2.02 & 2.88 & 3.52 & 2.52 \\
\hline
\end{tabular}

Cuadro 2. Modelo de regresión de efectos mixtos

\begin{tabular}{|l|c|c|c|c|c|c|c|c|c|}
\hline & & \multicolumn{10}{c}{ Semana } \\
\hline & $\mathbf{0}$ & $\mathbf{1}$ & $\mathbf{2}$ & $\mathbf{3}$ & $\mathbf{4}$ & $\mathbf{5}$ & $\mathbf{6}$ & $\mathbf{7}$ & $\mathbf{8}$ \\
\hline AFA & $\mathbf{4 . 1 0}$ & $\mathbf{3 . 7 0}$ & $\mathbf{4 . 0 0}$ & $\mathbf{3 . 5 7}$ & $\mathbf{4 . 0 5}$ & $\mathbf{2 . 0 5}$ & $\mathbf{3 . 9 0}$ & $\mathbf{3 . 5 2}$ & $\mathbf{2 . 5 2}$ \\
\hline Mañana & 4.43 & $\mathbf{5 . 0 0}$ & 4.29 & $\mathbf{3 . 1 4}$ & $\mathbf{3 . 7 1}$ & 2.43 & 4.14 & 4.14 & $\mathbf{3 . 4 3}$ \\
\hline Noche & $\mathbf{3 . 4 1}$ & 2.43 & $\mathbf{3 . 4 3}$ & $\mathbf{3 . 4 3}$ & 4.14 & 2.00 & $\mathbf{3 . 5 7}$ & 2.43 & 1.71 \\
\hline Tarde & 4.47 & $\mathbf{3 . 8 6}$ & 4.29 & 4.14 & 4.29 & 1.71 & 4.00 & 4.00 & $\mathbf{2 . 4 3}$ \\
\hline DOE & $\mathbf{4 . 5 1}$ & $\mathbf{3 . 8 9}$ & $\mathbf{3 . 1 7}$ & $\mathbf{2 . 7 1}$ & $\mathbf{3 . 3 5}$ & $\mathbf{2 . 0 0}$ & $\mathbf{1 . 8 6}$ & & \\
\hline Mañana & 4.51 & 4.67 & $\mathbf{3 . 8 3}$ & $\mathbf{3 . 1 4}$ & $\mathbf{3 . 6 7}$ & 2.57 & 2.57 & & \\
\hline Noche & 4.51 & 2.83 & 2.67 & $\mathbf{2 . 2 9}$ & 2.57 & 1.29 & 1.29 & & \\
\hline Tarde & 4.51 & 4.17 & $\mathbf{3 . 0 0}$ & $\mathbf{2 . 7 1}$ & $\mathbf{3 . 8 6}$ & 2.14 & 1.71 & & \\
\hline Tot & $\mathbf{4 . 3 1}$ & $\mathbf{3 . 7 9}$ & $\mathbf{3 . 6 2}$ & $\mathbf{3 . 1 4}$ & $\mathbf{3 . 7 1}$ & $\mathbf{2 . 0 2}$ & $\mathbf{2 . 8 8}$ & $\mathbf{3 . 5 2}$ & $\mathbf{2 . 5 2}$ \\
\hline
\end{tabular}


El Gráfico 1 presenta la evolución promedio según los resultados del modelo de regresión previamente estimado.

Gráfico 1. Evolución de dolor según tratamiento

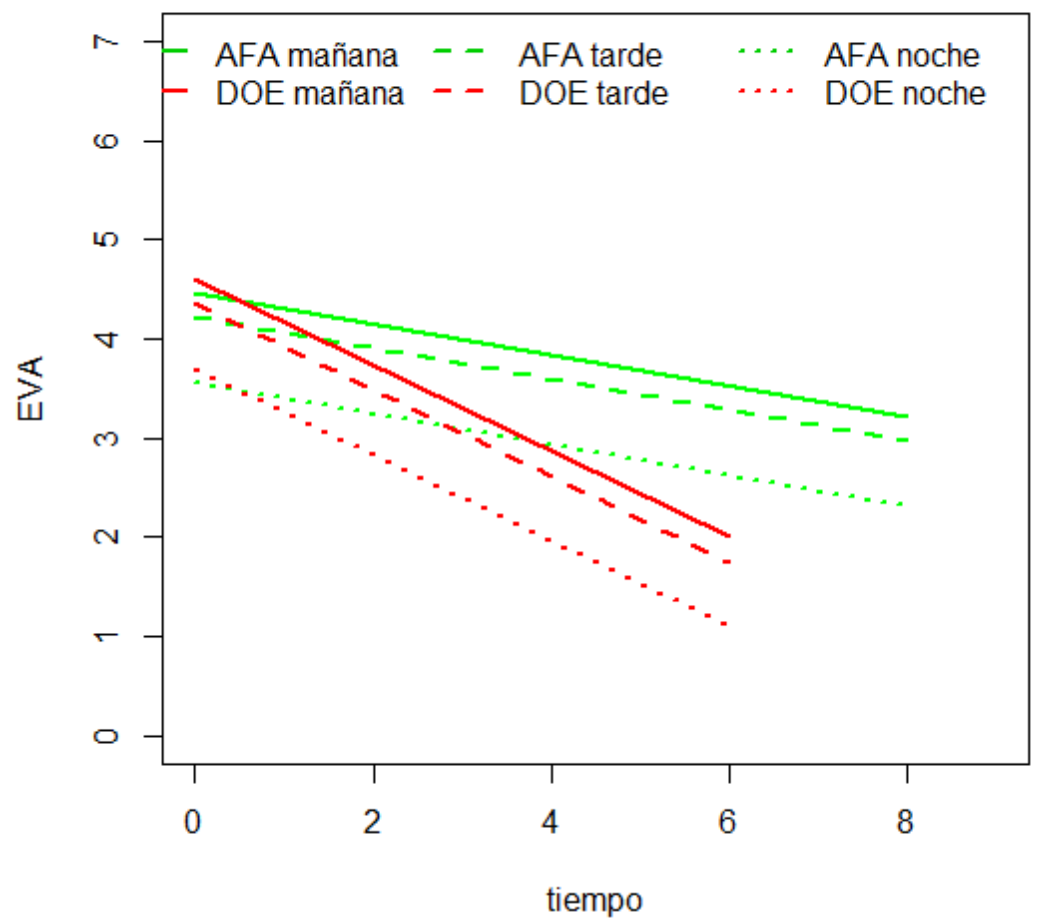

En el presente gráfico se puede observar que el descenso del tratamiento con DOE (líneas rojas) es más pronunciado que en las intervenciones con AFA (líneas verdes). Las tres líneas, correspondientes a cada tratamiento, responden a la evolución en los tres momentos del día, observándose que los valores de dolor en las noches son menores a los registrados en la mańana y tarde.

\section{Discusión}

De la lectura de las dos primeras líneas del Cuadro 2 se puede apreciar cómo el valor medio de dolor de ambos grupos no fue significativamente distinto, siendo el promedio 4.463 puntos. La tercera línea presenta el primer resultado relevante, el cual indica que el tratamiento AFA se caracterizó por un descenso semanal significativo de 0.155 puntos en la medición del do- lor. Complementando la afirmación anterior, la última fila muestra que en el caso del tratamiento con DOE, la tendencia fue aún más pronunciada que en el tratamiento con AFA, siendo esta diferencia significativa. Al combinar ambas estimaciones se obtiene que el descenso en la medición del dolor del grupo DOE fuera casi medio punto (0.434) por semana.

Adicionalmente se pudo constatar que el dolor percibido por los participantes fue menos intenso (casi un punto de EVA) en la noche con respecto a la mańana y que los eventos agravantes registrados podrían ser considerados significativos, teniendo un efecto tal que cuando los participantes presentaron dichos eventos, su percepción de dolor fue en promedio 0.794 puntos mayor a lo habitual.

En este estudio se encontraron diferencias significativas entre ambos grupos tras las interven- 
ciones en la variable dolor. Si bien con la AFA disminuyó el dolor significativamente, con los DOE fue con mayor tendencia y la diferencia en la disminución entre ambos grupos fue significativa. El período de evaluación del grupo tratado con DOE fue de 6 semanas, en este estudio se puede apreciar su efectividad para disminuir el síntoma dolor. Este resultado se alinea con estudios como el de Dao y Lavigne 1994 que demuestran la disminución del síntoma dolor en un tiempo de evaluación de 10 semanas, pero sin explicar el mecanismo de disminución del mismo ${ }^{(8)}$. Más adelante Kreiner en una revisión de la literatura concluía que con los datos disponibles hasta ese momento el uso de DOE para el dolor muscular y articular tenia soporte científico suficiente ${ }^{(9)}$. En 2011 un estudio clínico randomizado que comparo la eficacia de los DOE con terapia física (Tens) para el control de dolor por TTM en un periodo de evaluación para los DOE de 4 meses y para la modalidad de tratamiento físico de 4 semanas, concluyó que existe una diferencia significativa en la disminución del dolor entre ambas modalidades estudiadas, siendo los DOE los más efectivos ${ }^{(20)}$. Este último estudio se alinea con más estudios de la eficacia de los DOE en el tratamiento de dolor por periodos de 5 semanas (21) y 10 semanas respectivamente ${ }^{(22)}$.

Nuestro trabajo está en concordancia con los estudios revisados y a pesar de existir controversia en cuanto al modo o mecanismo de acción, los DOE son, efectivamente, dispositivos que disminuyen el dolor músculo esqueletal en los pacientes con TTM y a pesar de los tiempos variados de evaluación su eficacia es reconocida. La AFA tuvo una tendencia a disminuir más lentamente que el DOE, esto podría deberse a que los voluntarios eran personas sedentarias y en la AFA fue incrementando su intensidad al pasar los días de intervención. Es necesario tener intensidad de la AFA para asegurarse la liberación de $\mathrm{BE}{ }^{(14)}$. Existen estudios que demostraron que los niveles de $\mathrm{BE}$ no variaron con intensidades de ejercicio bajas, con volú- menes de oxigeno que variaron entre el 25\% y el $60 \%{ }^{(23,24,25)}$.

Otro estudio sobre 12 hombres no entrenados, se observó que pedaleando a $60 \%$ de volumen de oxigeno no ocurrieron cambios significativos en los niveles de BE. Sin embargo, en intensidades de 70 y $80 \%$, los aumentos en los niveles fueron significativos de 2 a 5 veces a los valores de base, respectivamente ${ }^{(26)}$. El mismo autor, años después, no encontró diferencia entre género en cuanto a los niveles de $\mathrm{BE}$ en diferentes intensidades de ejercicio aeróbico 60 y $80 \%$ respectivamente ${ }^{(27)}$. Uno de los parámetros más difícil de estimar es la intensidad de la actividad física. Hay diferentes métodos para medirla, medir la frecuencia cardiaca, apreciación del practicante en relación a su actividad y atribución de intensidad utilizando un sistema de clasificación uniforme ${ }^{(28)}$. En nuestro estudio no se midió el volumen de oxígeno en forma directa sino que fue a través de la fórmula de Karvonen ${ }^{(19)}$. Tampoco tenía como objetivo la medición de BE.

El artículo de revisión de Nishishiya en 2006, analizó la eficacia de AFA en individuos con fibromialgia y pudo constatar que la AFA son los ejercicios más estudiados y se concluyó que existe evidencia moderada de que AFA produce mejoría del dolor en los pacientes estudiados. Así mismo no existe evidencia alguna de que la AFA empeore las manifestaciones clínicas de los individuos con fibromialgia ${ }^{(29)}$.

Más reciente un artículo de revisión publicado en 2017 se habla de que la calidad de la evidencia que examina la actividad física y el ejercicio para el dolor crónico es baja. Esto se debe en gran parte a tamaños de muestra pequeños y estudios potencialmente poco potentes. Se detectaron efectos favorables en la reducción de la gravedad del dolor y la mejora de la función física, aunque estos fueron principalmente de efecto bajo a moderado y no fueron consistentes en todas las revisiones ${ }^{(30)}$. En este sentido nuestro estudio tendría los mismos efectos que se relatan en ese artículo en cuanto al síntoma del dolor. 
La evidencia disponible sugiere que la actividad física y el ejercicio es una intervención con pocos eventos adversos que pueden mejorar la intensidad del dolor, la función física, y la consiguiente calidad de vida. Sin embargo, se requiere más investigación y debería centrarse en aumentar el número de participantes, incluidos los participantes con un espectro más amplio del nivel EVA del dolor, y alargar, tanto la intervención en sí como el período de seguimiento ${ }^{(30)}$.

\section{Conclusiones}

El presente estudio demostró que tanto la intervención con DOE como con AFA contribuyeron a la disminución del dolor orofacial de origen músculo esqueletal en voluntarios con TTM.

El estudio comparativo entre estos tratamientos demostró que la eficacia en la disminución del dolor fue más efectiva en el grupo con DOE.

Futuros estudios deberían revisar y analizar la posibilidad de otros diseños metodológicos que aporten más precisión a los resultados.

Periodos de evaluación más prolongados, muestras de mayor tamaño, intervenciones de AFA más homogéneas.

También diseños en que a los 2 grupos se les instale el DOE y a uno de ellos se le sume la AFA podría ser interesante; así mismo la posibilidad de conformar 3 grupos: uno con DOE, otro con AFA y un tercero con DOE y AFA.

\section{Contribución de autoría}

1. Concepción y diseño del estudio

2. Adquisición de datos

3. Análisis de datos

4. Discusión de los resultados

5. Redacción del manuscrito

6. Aprobación de la versión final del manuscrito

LARF ha contribuido en $1,2,3,4,5$ y 6 . LS ha contribuido en 2, 3 y 4 .

RRB ha contribuido en 1, 3, 4 y 6 .

\section{Referencias}

1. Okeson JP. Tratamiento de la oclusión y afecciones temporomandibulares. $7^{\mathrm{a}} \mathrm{ed}$. Barcelona: Elsevier, 2013. p102-128.

2. GPT-9 Glossary of Prosthodontics Terms, Edition 9, J Prosthet Dent 2017; Vol.117: 1-106

3. Le Resche L. Epidemiology of Temporomandibular Disorders: Implications for the Investigation of Etiologic Factors. Crit Rev Oral Biol Med. 1997; 8 (3): 291-305. DOI: $10.1177 / 10454411970080030401$

4. Jussila P, Kiviahde H, Näpänkangas R. Prevalence of Temporomandibular Disorders in the Northern Finland Birth Cohorts 1966. J Oral Facial Pain Headache. 2017; 31: 159-164. doi: 10.11607/ofph.1773

5. Lung J, Bell L, Heslop M, Cuming S, Ariyawardana A. Prevalence of Temporomandibular disorders among a cohorts of university undergraduates in Australia. J Invest Clin Dent. 2018; 1-5 dos.org/10.1111/jicd.12341.

6. Riva R., Sanguinetti M, Rodriguez A, Guzzetti L, Lorenzo S. Prevalencia de trastornos temporomandibulares y bruxismo en Uruguay Parte 1. Odontoestomatología. 2011; 8 (17): 54-71.

7. Michelotti A, De Wijer A, Steenks M, Farella M. Home-exercise regimes for the management of non-specific temporomandibular disorders. Journal of Oral Rehabilitation. 2005; 32: 779785.

8. Dao T, Lavigne G, Charbonneau A. The efficacy of oral splints in the treatment of myofascial pain of the jaw muscles: a controlled clinical trial. Pain. 1994; 56: 85-94.

9. Kreiner M, Bentancor E, Clark G. Occlusal stabilization appliance. Evidence of their efficacy JADA. 200; 132: 770-777.

10. Lopez-Rodriguez M, Castro-Sanchez A, Fernandez-Martinez M. Comparacion entre biodanza en medio acuático y stretching en la mejora de la calidad de vida y dolor en los pacientes con fibromialgia. Aten Primaria. 2012; 44(11): 641-650.

11. Valim V, Natour J, Xiao Y. Efeitos do exercício fisico sobre os níveis sericos de serotonina e seu metabolito na fibromialgia: um estudio piloto randomizado. Rev. Bras Reumatol. 2013; 53 (6): $538-541$ 
12. Borja Sañudo, Gailano D, Carrasco L. Efects of a prolonged exercise program on key health outcomes in women with fibromyalgia: A randomized controlled trial. J. Rehabil Med. 2011; 43: 521-526.

13. Fontaine K, Conn L, Clauw D. Effects of Lifestyle Physical Activity in Adults with Fibromyalgia: Results at Follow-up. J Clin Rheumatol. 2011; 17 (2): 64-68. doi: 10.1097/ RHU.0b013e31820e7ea7.

14. Cunha G, Ribeiro J, Oliveira A. Níveis de BetaEndorfina em Resposta ao Exercício e no Sobretreinamento. Arq Boas Endocrinol Metab. 2008; 52 (4): 589-598.

15. Schwarz L, Kinderman W. Cannes in B- Endorphin levels in response to aerobic an anaerobic exercise. Sports Medicine. 1992; 13 (1): 25-36

16. Shiffman E, Ohrbach R, Truelove E. Diagnostic Criteria for Temporomandibular Disroders (DC/TMD) for clinical and Research Applications: recommendations of the International RDC/TMD Consortium Network* and Orofacial Pain Special Interest Group. J Oral Facial Pain Headache. 2014; 28 (1): p6-27.

17. Gonzalez F, Guardia L, Schiavone G,. Programa de ejercicios aerobicos o de resistencia cardiorespiratoria. En Mazza M. Manual para la prescripción de ejercicio: actividad física y ejercicio para la salud. 1era. ed. Montevideo, Cátedra de Medicina del ejercicio y deporte: DEDOS; 2011, p129-165.

18. Deppiésse F. La prescription des activités physiques en practique médicale courante. En: Depiésse F, Coste O. Prescription des activités physiques en prévention thérapeutique. $2^{\mathrm{a}}$ edition Issy-les-Moulineaux: Elsevier, 2016, p129165.

19. Karvonen J, Vourimaa T. Heart rate and exercice intensity during sports activities. Practical application. Sports Med.1988; 5 (5): p303-11.

20. Azam S. Madani, Amirtaher Mirmotazavi. Comparison of three treatment options for painful temporomandibular joint clicking. Journal of Oral Science. 2011; 53 (3): 349-354.
21. Vicente-Barrero M, Yu-Lu S., Zhang B. The efficacy of acupunture and decompression splints in the treatment of temporomandibular joint pain-dysfunction syndrome. Med Oral Patol Oral Cir Bucal. 2012. 17 (6): 1028-1033.

22. Ficnar T, Middelberg C, Rademacher B. Evaluation of the effectiveness of a semi-finished occlusal aplaiance- a randomized, controlled clinical trial. Head\&Face Medicine- 2013; 9: 5 http: //www.head-face-med.com/content/9/1/5.

23. Langenfeld M, Hart L, Kao P. Plasma B-endorphin responses to one-houre bicycling and running at 60\% O2 max. Medicine\&Science in Sports\&Exercice. 1987; 19 (2): 83-86.

24. Rahkila P, Hakala E, Salminen K. Response of plasma endorphin to running exercises in male and female endurance athletes. Med Sci Sports Exerc. 1987; 19 (5): 451-5.

25. Maresh C, Sökmen B, Kraemer W. Pituitaryadrenal responses to arm versus leg exercise in untrained man. Eur. J Appl Physiol 2006. 97: 471-477.

26. Goldfarb A, Hatfield B, Potts J. Beta-endorphin time course response to intensity of exercise: effects of training status. Int J Sports Med. 1991; 12 (3): 264-8

27. Golfarb A., Jamurtas A., Kamimori G. Gender effects on beta-endorphin response to exercise. Med Sci Sports Exerc. 1998; 30 (12): 1672-6.

28. Hayot, M. La réhabilitation du malade respiratoire chronique. En Préfaut C. y Ninot G. Les test d'exercice: lépreuve dexercice, a charge croissant.Issy les Moulineaux: Elsevier Masson, 2009; p86-112.

29. Nishishinya M, Rivera J, Cayetano A. Intervenciones no farmacológicas y tratamientos alternativos en la fibromialgia. Med Cin (Barc). 2006; 127 (8): 295-9.

30. Geneen LJ, Moore RA, Clarke C, Martin D, Colvin LA, Smith BH. Physical activity and exercise for chronic pain in adults: an overview of Cochrane Reviews. Cochrane Database of Systematic Reviews. 2017; 1 (1): CD011279.

\section{Luis Andrés Rodríguez Figueroa: anrodent@yahoo.com}

